Original Article

\title{
The varying effects of warm-water bathing therapies: partial bathing decreases exercise tolerance to levels similar to full-body bathing
}

\author{
Tadasu Ohshige, RPT, PhD ${ }^{1)^{*}}$, Aкiniko Ohwatashi, RPT, PhD ${ }^{1)}$, Ryoj Kiyama, RPT, PhD $^{1)}$ \\ 1) Course of Physical Therapy, School of Health Sciences, Faculty of Medicine, Kagoshima University: \\ 8-35-1 Sakuragaoka, Kagoshima 890-8544, Japan
}

\begin{abstract}
Purpose] The purpose of this study was to determine differences in postural sway and tolerance to exercise before and after full-body, forearm, and lower leg bathing in warm-water. [Subjects and Methods] Thirteen healthy, young adult males were subjected to full-body, forearm, and lower leg bathing at $41{ }^{\circ} \mathrm{C}$ for 10 minutes. [Results] The 2-point discrimination sense value and total trajectory length significantly decreased after bathing. [Conclusion] In summary, we found that warm-water bathing sharpens plantar sensation, and thus may help to prevent falls in the elderly. Even partial forearm and lower leg bathing increased exercise tolerance to levels similar to full-body bathing.

Key words: Warm-water bathing, Static balance, Exercise test
\end{abstract}

(This article was submitted Jun. 24, 2015, and was accepted Aug. 3, 2015)

\section{INTRODUCTION}

Murata $^{1)}$ demonstrated that stronger toe foot-gripping and sharper plantar sense decreased postural sway. Sakita et al. ${ }^{2)}$ showed that cooling the lower leg decreased plantar sense, increasing postural sway. However, to the best of our knowledge, no previous studies have investigated the effect of warm-water bathing, including full-body, forearm, and lower leg bathing, on postural sway. Furthermore, there are no studies on the effect of warm-water bathing on exercise tolerance. Here, we evaluated the effects of full-body, forearm, and lower leg bathing on vital reaction, 2-point discrimination sense, postural sway, and exercise tolerance.

\section{SUBJECTS AND METHODS}

We recruited thirteen healthy, young, male participants (13 males; mean age \pm standard deviation (SD), $20.3 \pm$ 1.3 years). Measurements were collected at room temperature (approximately $22^{\circ} \mathrm{C}$ ) and in bath water heated to $41^{\circ} \mathrm{C}$. Three bathing methods were used: full-body bathing, forearm bathing, and lower leg bathing. The bathing duration was 10 minutes. Each bathing method was implemented randomly on different dates. The vital reaction was measured during bathing by collecting the sublingual temperature (CM-210 Terumo Co., Ltd., Tokyo, Japan), heart rate (BSM-

*Corresponding author. Tadasu Ohshige (E-mail: ohshige@ health.nop.kagoshima-u.ac.jp)

C2015 The Society of Physical Therapy Science. Published by IPEC Inc. This is an open-access article distributed under the terms of the Creative Commons Attribution Non-Commercial No Derivatives (by-ncnd) License $<$ http://creativecommons.org/licenses/by-nc-nd/3.0/>.
2401 Nihon Koden Co., Ltd., Tokyo, Japan), blood pressure, and respiratory rate. Each measurement was collected during rest before bathing (pre-bath) and at 10 minutes after the bath was completed (post-bath). In order to assess the heat insulation state, the sublingual temperature was also measured 30 minutes post-bath. The 2-point discrimination sense value and the postural sway were measured before bathing and at 30 minutes post-bath. The 2-point discrimination sense was measured at the thenar eminence on the side of the pivot leg and the hallux. To determine the postural sway, the participants were asked to stand on one leg (the pivot leg) for one minute. Sampling frequence was $30 \mathrm{~Hz}$, above the force platform (PDM, zebris Medical $\mathrm{GmbH}$, Isny, Germany). This test was performed three times, and the postural sway was calculated by averaging the total trajectory length of the three trials. The exercise test was performed before bathing and at 30 minutes post-bath using a bicycle ergometer. The metabolic equivalents (Mets) score was calculated based on the oxygen consumption measured using an oxygen consumption meter (AT-1100 Anima Co., Ltd., Tokyo, Japan).

This study was approved by the Clinical Review Board of the Faculty of Medicine at Kagoshima University (No. 284). All the participants signed a consent form after being informed of the study purpose and content, and all risks involved.

All statistical calculations were performed using SPSS 23 software. The Shapiro-Wilk test was used to evaluate the normal distribution of the data. The measurement time points and the bathing methods were analyzed using a 2-way repeated-measures ANOVA. Regarding our factors, we used Mauchly's test of sphericity to assess the degree of significance between the two levels. When interactions between three factor levels produced a p-value $<0.05$ and 
Table 1. Two-way repeated measures ANOVA and multiple comparisons for each bathing method and their experimental variables

\begin{tabular}{|c|c|c|c|c|}
\hline Experimental variables & & Full-body bathing & Forearm bathing & Lower leg bathing \\
\hline \multirow{3}{*}{ Sublingual temperature $\left({ }^{\circ} \mathrm{C}\right)$} & Before bathing & $36.6 \pm 0.3$ & $36.6 \pm 0.3$ & $36.7 \pm 0.1$ \\
\hline & 10 min after bathing & $37.5 \pm 0.2^{\#}$ & $37.0 \pm 0.3^{\#}$ & $36.8 \pm 0.2^{\# *}$ \\
\hline & 30min after bathing & $36.8 \pm 0.2$ & $36.7 \pm 0.3$ & $36.7 \pm 0.2$ \\
\hline \multirow{2}{*}{ Heart rate (bpm) } & Before bathing & $77.2 \pm 7.2$ & $75.4 \pm 8.2$ & $73.9 \pm 9.1$ \\
\hline & 10 min after bathing & $96.3 \pm 8.4^{\#}$ & $82.9 \pm 8.6^{\# *}$ & $79.3 \pm 9.3^{\# *}$ \\
\hline \multirow{2}{*}{ Systolic blood pressure (mmHg) } & Before bathing & $126.6 \pm 10.8$ & $123.2 \pm 9.5$ & $125.4 \pm 15.4$ \\
\hline & 10 min after bathing & $114.5 \pm 8.8$ & $125.0 \pm 16.1$ & $123.3 \pm 14.3$ \\
\hline \multirow{2}{*}{ Diastolic blood pressure (mmHg) } & Before bathing & $77.4 \pm 6.2$ & $74.9 \pm 6.3$ & $77.8 \pm 8.9$ \\
\hline & 10 min after bathing & $55.7 \pm 5.4^{\#}$ & $73.6 \pm 8.6^{\# *}$ & $78.7 \pm 10.8^{\# \star}$ \\
\hline \multirow{2}{*}{ Respiratory rate (Trial/min) } & Before bathing & $17.5 \pm 4.8$ & $17.2 \pm 4.5$ & $18.2 \pm 2.6$ \\
\hline & 10 min after bathing & $17.54 \pm 5.6$ & $18.9 \pm 3.0$ & $19.9 \pm 4.0$ \\
\hline \multirow{2}{*}{$\begin{array}{l}\text { 2-point discrimination sense value } \\
\text { for thenar eminence }(\mathrm{mm})\end{array}$} & Before bathing & $8.0 \pm 2.4$ & $8.6 \pm 2.8$ & $7.9 \pm 2.0$ \\
\hline & 30 min after bathing & $6.2 \pm 2.1^{\#}$ & $7.7 \pm 2.6^{\#}$ & $6.5 \pm 1.6^{\#}$ \\
\hline \multirow{2}{*}{$\begin{array}{l}\text { 2-point discrimination sense value } \\
\text { for hallex }(\mathrm{mm})\end{array}$} & Before bathing & $7.9 \pm 2.4$ & $8.2 \pm 2.3$ & $8.1 \pm 2.2$ \\
\hline & 30 min after bathing & $6.8 \pm 2.0^{\#}$ & $6.7 \pm 2.5^{\#}$ & $6.9 \pm 1.8^{\#}$ \\
\hline \multirow{2}{*}{ Total trajectory length (mm) } & Before bathing & $1131 \pm 218$ & $1151 \pm 271$ & $1183 \pm 202$ \\
\hline & 30 minutes after bathing & $998 \pm 183^{\#}$ & $1011 \pm 224^{\#}$ & $982 \pm 149^{\#}$ \\
\hline \multirow{2}{*}{ MetS score for $25 \mathrm{~W}$} & Before bathing & $2.4 \pm 2.1$ & $2.3 \pm 0.4$ & $2.3 \pm 0.3$ \\
\hline & 30 minutes after bathing & $2.1 \pm 0.3^{\#}$ & $2.1 \pm 0.3^{\#}$ & $2.0 \pm 0.3^{\#}$ \\
\hline
\end{tabular}

${ }^{\#}$ Compared to pre-bath values $(\mathrm{p}<0.05)$

*Compared to full-body bathing values $(\mathrm{p}<0.05)$

a statistically significant result from the Mauchly's test of sphericity, we performed a Bonferroni's multiple comparison test. Bonferroni post hoc test correction was applied during the multiple comparison tests.

\section{RESULTS}

At the 10 minutes post-bath time point, the sublingual temperature was significantly increased compared with the pre-bath temperature under all bathing conditions. Compared to lower leg bathing, the full-body bathing significantly increased sublingual temperature by $0.9^{\circ} \mathrm{C}$ (Table 1 ). Although the average sublingual temperature at 30 minutes post-bath was increased under all bathing conditions, the data were not statistically significant. The heart rate at 10 minutes post-bath was significantly increased $(\mathrm{p}<0.05)$ compared with the pre-bath values under all bathing conditions. The heart rate was particularly heightened during fullbody bathing, increasing by $19.1 \mathrm{bpm}(\mathrm{p}<0.05)$, whereas forearm and lower leg bathing increased the rate $7.5 \mathrm{bpm}(\mathrm{p}$ $<0.05)$ and $5.4 \mathrm{bpm}(\mathrm{p}<0.05)$, respectively. Systolic blood pressure decreased by $12.1 \mathrm{mmHg}$ in full-body bathing compared with pre-bath measurements, although the difference between the pre-bath and 10 minutes post-bath values was not significant. Moreover, a significant decrease in diastolic blood pressure was observed when comparing pre-bath and 10 minutes post-bath values for full-body and forearm bathing. Full-body bathing demonstrated a more significant decrease in diastolic blood pressure than forearm bathing based on the p-value. The values dropped dramatically from $77.4 \mathrm{mmHg}$ to $55.7 \mathrm{mmHg}$, a $21.7 \mathrm{mmHg}$ decrease.

The respiratory rate was not significantly different between pre-bath and 30 minutes post-bath. The 2-point discrimination sense significantly decreased 30 minutes post-bath compared with pre-bath values. Consequently, the 2-point discrimination results indicate that legs become sensitive after bathing. Meanwhile, the total trajectory length significantly decreased 30 minutes post-bath. The largest decrease was seen in lower leg bathing. The total trajectory length decreased from 1,183 $\mathrm{mm}$ to $982 \mathrm{~mm}$, a $201 \mathrm{~mm}$ decrease. Thus, these results demonstrate that the postural sway decreases after warm-water bathing. During exercise tolerance assessments, the Mets score decreased by 0.3 Mets following full-body bathing, 0.2 Mets following forearm bathing, and 0.3 Mets following lower leg bathing.

\section{DISCUSSION}

We selected a $41{ }^{\circ} \mathrm{C}$ bathwater temperature based on the report by Horikiri ${ }^{3)}$ that suggested the optimal bathing temperature for people with cardiovascular disease is between 40 to $41{ }^{\circ} \mathrm{C}$. Anything above that temperature range runs the risk of overloading the patients' hearts. In our study, fullbody bathing induced potent vasodilation and produced the largest heart rate increase. This is consistent with Tanaka ${ }^{4)}$ who demonstrated that heart rate increases are induced by reflective sympathicotonia and inhibition of the vagus nerve due to vasodilation and decreased blood pressure. Our data showed that warm-water bathing increased 2-point discrimination sensitivity and decreased the total trajectory length of the postural sway, which led to static balance. In Tanaka's study $^{5,6)}$, the older patients showed a significant decrease in the patient's sense of the toe compared with their younger counterparts. Similarly, static balance was less pronounced 
among older adults than young adults ${ }^{7)}$. These results are consistent with $\mathrm{Cho}^{8)}$ who demonstrated that falls in poststroke patients were associated with reduction in static balance. Ikeda ${ }^{9}$ ) showed immediate improvement in standing balance among frail older patients with lumbar spinal canal stenosis following thermotherapy. Thus, strategic implementation of warm-water bathing to sharpen plantar sense in elderly patients may prevent potentially devastating falls.

Horikiri ${ }^{10)}$ argued that the increase in exercise tolerance observed following warm-water bathing can be attributed to a heat-induced improvement in overall blood flow, including coronary vessels, and enhanced musculoskeletal movement due to collagen softening. Furthermore, Kihara ${ }^{11)}$ reported that improved expanded heart function may be an acute effect of body heating. Thus, our Mets score decrease, observed during the exercise tolerance test, might have resulted from diminished heart load caused by the heightened body temperature. The body temperature increase may have contributed to a decline in the heart preload and a decline in the heart afterload induced by telangiectasia function.

Initially we had assumed that full-body bathing would demonstrate larger impact on exercise tolerance. However, our decreased Mets values during forearm and lower leg bathing were similar to those of full-body bathing. Therefore, the results of our study demonstrated that even partial bathing, such as forearm bathing and lower leg bathing, can increase exercise tolerance to levels similar to full-body bathing.

\section{REFERENCES}

1) Murata S: Relationship between body sway of one-leg standing with vision and foot function in healthy female. Rigakuryoho Kagaku, 2004, 19: 245-249. [CrossRef]

2) Sakita M, Kumagai $S$, Kawano I, et al.: Standing postural control after cooling of sole and crural Muscles -Comparisons of muscle activities and lengths of center of gravity sway on static and dynamic postural control with eyes closed-. Rigakuryoho Kagaku, 2006, 21: 341-347. [CrossRef]

3) Horikiri $Y$, Tai C, Tanaka N: Circulatory changes by hot bathing. Gen Rehabil, 1991, 19: 1057-1061.

4) Tanaka N, Hiyoshi T, Takesako K, et al.: Effects of artificial mineral spring (Basukurin) on hemodynamic functions, deep body temperature, autonomic nervous functions and blood gas concentration. J Jpn Soc Balneol Climatol Phys Med, 1987, 50: 187-196.

5) Tanaka T, Noriyasu S, Ino $S$, et al.: Objective method to determine the contribution of the great toe to standing balance and preliminary observations of age-related effects. IEEE Trans Rehabil Eng, 1996, 4: 84-90. [Medline] [CrossRef]

6) Tanaka T, Hashimoto N, Nakata M, et al.: Analysis of toe pressures under the foot while dynamic standing on one foot in healthy subjects. J Orthop Sports Phys Ther, 1996, 23: 188-193. [Medline] [CrossRef]

7) Maeda Y, Tanaka T, Miyasaka T, et al.: A preliminary study of static and dynamic standing balance and risk of falling in an independent elderly population with a particular focus on the limit of stability test. J Phys Ther Sci, 2011, 23: 803-806. [CrossRef]

8) Cho K, Yu J, Rhee H: Risk factors related to falling in stroke patients: a cross-sectional study. J Phys Ther Sci, 2015, 27: 1751-1753. [Medline] [CrossRef]

9) Ikeda T, Kugota H, Oya N, et al.: Effect of thermal therapy of the feet on balance function for elderly persons with lumber spinal canal stenosis. Jpn J Electrophysical Agents, 2014, 21: 75-78.

10) Horikiri $Y$, Himodozono $M$, Wang $X J$, et al.: Improvement of exercise tolerance after hot water bating in aged men. J Jpn Soc Balneol Climatol Phys Med, 2000, 63: 138-142.

11) Kihara T, Tei $C$ : Waon therapy in patients with dilated cardiomyopathy. Heart Views, 2008, 12: 1000-1005. 